\title{
Suspected malignant biliary strictures: from inside out or outside in?
}

\section{다 (ㄱ) $\odot$}

\author{
Author \\ Frederic Prat \\ Institution \\ Paris-Descartes University, Assistance Publique-Hôpitaux \\ de Paris, Paris, France \\ Bibliography \\ DOI https://doi.org/10.1055/s-0044-101353 | \\ Endoscopy International Open 2018; 06: E778-E779 \\ (c) Georg Thieme Verlag KG Stuttgart · New York \\ ISSN 2364-3722
}

Biliary strictures, inducing cholestasis and jaundice, result from an obstructive process that can emerge intraluminally from the biliary epithelium, either a cholangitis or a primary cholangiocarcinoma, or extraluminally from compression by a pancreatic malignancy, a metastatic mass or a regional inflammatory process such as pancreatitis. The list of diseases, benign and malignant, which can produce a biliary stenosis is pretty long, although $80 \%$ are malignant, with pancreatic carcinoma the first in line $[1,2]$. However, the diagnosis is often easy when a mass is clearly defined and amenable to endoscopic ultrasound-guided fine-needle aspiration (EUS-FNA), which has nearly $90 \%$ sensitivity [3]. The diagnosis is also straightforward when orthotopic liver transplantation is complicated by a short stricture centered on the bilio-biliary anastomosis, or after a difficult cholecystectomy with bile duct injury and protracted biliary leak. However, when the mass is missing and the clinical history is poor or unclear, the stricture can remain indeterminate for a while, which places physicians in the uncomfortable position of operating for a benign disease with potentially undue morbidity and mortality or leaving an undiagnosed malignancy unchecked. Diagnosis in these cases relies mostly on endoscopic retrograde cholangiopancreatography (ERCP)-based sampling techniques, essentially brush cytology and/or fluoroscopyguided forceps biopsies, which have a sensitivity of less than $50 \%[2,4]$.

In this issue of Endoscopy International Open, Moura et al. have tried to improve our understanding of the respective inputs of both approaches by comparing the diagnostic performances of ERCP-guided sampling and EUS-FNA in a group of 50 patients selected for a suspected biliopancreatic malignancy revealed by a biliary stricture. The patient selection process, although claiming only indeterminate strictures were included, understates that most patients had a tissular mass obstructing the bile duct which was amenable to EUS-FNA, after which one can predict that most strictures will not remain indeterminate. However, the strength of the study was to have each patient be his or her own control by performing both EUS-FNA and ERCP sampling during the same sedation. Sampling methods were state-of-the-art, including both suction and the slow-pull capillary method for FNA, and a combination of brushing and biopsies during ERCP with cell-block technique to optimize cytological diagnosis. The authors based the study power on a reasonable hypothesis of a $75 \%$ vs $49 \%$ difference of sensitivity in favor of EUS-FNA. Surprisingly, after surgical resection or a follow-up of at least 6 months, only 2 out of 50 patients (4\%) were found to have benign disease, meaning that patients were highly selected before enrollment with a very strong suspicion of malignancy. Sensitivities for ERCP-guided techniques were, as expected, much lower than that for EUS-FNA, with $61 \%$ for the combined brushing and fluoroscopy-guided biopsies, and short of $40 \%$ for brushings alone, as against nearly $94 \%$ for EUS, whereas the combination of ERCP and EUS slightly increased overall sensitivity to close to $98 \%$. However, as a consequence of the super selection of patients, negative predictive values were very low, below $10 \%$ for ERCP, at only $40 \%$ for EUS and no more than $67 \%$ for combined EUS and ERCP, a figure closer to what is expected for EUS alone in an unselected patient population with a solid pancreatic mass [3]. The proximal vs distal location of the stricture did not appear to significantly affect the diagnostic performance of either technique, although EUS did slightly better in distal rather than proximal strictures and ERCP did the opposite. A more important finding was that the superiority of EUS was overwhelming in bigger lesions, here considered as larger than $1.5 \mathrm{~cm}$ in diameter, whereas both methods were rated equally in smaller lesions, at $50 \%$ sensitivity, although the figure was probably based on a small number 
of cases. Finally, the superiority of EUS was once again clearly demonstrated in extraductal lesions (ie pancreatic carcinomas) but not so much in intraductal ones (ie primary biliary carcinomas) with an accuracy of $82.4 \%$ for EUS vs. $70.6 \%$ for ERCP.

The weaknesses of ERCP-guided sampling techniques are well-known: whereas extraductal masses, generally pancreatic or lymphatic, cannot be adequately sampled from a neighboring structure unless they invade its lumen, the superficial scratching of intraductal strictures is also frequently inconclusive because those lesions, whether inflammatory or neoplastic, generally develop a thick and dense fibrous microenvironment within which the cells of interest can be scarce and/or deeply seated [5]. Even fluoroscopy-guided biopsies, although reaping deeper material, are often unsuccessful because targeting is inaccurate and the tangential incidence of the forceps jaws makes biopsies too superficial. On the contrary, EUS-FNA or endoscopic ultrasound-guided fine-needle biopsy (EUSFNB) is excellent at collecting rich cytology and even microbiopsies, especially with recent refinements in needle designs and sampling techniques [6,7], but that comes with a caveat: EUS-FNA/FNB requires a target volume, approximately over $500 \mathrm{~mm}^{3}$ or $10 \mathrm{~mm}$ in diameter, to be efficient. When the target is too discrete, very thin and purely infiltrative, EUS results rapidly decrease and lead to stricture indetermination. In such cases, ERCP brushings and biopsies remain the first-line diagnostic option, but still leave about $50 \%$ of cases indeterminate. This is the place for more advanced techniques, such as ERCPguided retrograde cholangioscopy with endoscopically, not fluoroscopically, guided-biopsies, which can solve a significant part of the problem of indeterminate biliary strictures [8]. The diagnostic performance of cholangioscopy in such cases has been estimated at about $70 \%$ in recent meta-analyses with single-operator devices $[9,10]$, but the impact on patient management and outcomes remains to be assessed. The residual $30 \%$ of indeterminate biliary strictures will have to be dealt with using either innovative sampling methods or more advanced, molecular-based analyses to overcome current cytological limitations [11, 12].

\section{Competing interests}

None

\section{References}

[1] Singh A, Gelrud A, Agarwal B. Biliary strictures: diagnostic considerations and approach. Gastroenterology Report 2015: $22-31$

[2] Tamada K, Ushio J, Sugano K. Endoscopic diagnosis of extrahepatic bile duct carcinoma: Advances and current limitations. World J Clin Oncol 2011; 2: 203-216

[3] Puli SR, Bechtold ML, Buxbaum JL et al. How good is endoscopic ultrasound-guided fine-needle aspiration in diagnosing the correct etiology for a solid pancreatic mass? A meta-analysis and systematic review Pancreas 2013; 42: 20-26

[4] Navaneethan U, Njei B, Lourdusamy V et al. Comparative effectiveness of biliary brush cytology and intraductal biopsy for detection of malignant biliary strictures: a systematic review and meta-analysis. Gastrointest Endosc 2015; 81: 168 -176

[5] Banales JM, Cardinale V, Carpino G et al. Cholangiocarcinoma: current knowledge and future perspectives consensus statement from the European Network for the Study of Cholangiocarcinoma (ENS-CCA). Nature Rev Gastroenterol Hepatol 2016; 13: 261 - 280

[6] Wani S, Muthusamy VR, Komanduri S. EUS-guided tissue acquisition: an evidence-based approach (with videos). Gastrointest Endosc 2014; 80: 939

[7] Alatawi A, Beuvon F, Grabar S et al. Comparison of 22G reverse-beveled versus standard needle for endoscopic ultrasound-guided sampling of solid pancreatic lesions. United European Gastroenterol J 2015; $3: 343-352$

[8] Navaneethan U, Hasan MK, Lourdusamy V et al. Single-operator cholangioscopy and targeted biopsies in the diagnosis of indeterminate biliary strictures: a systematic review. Gastrointest Endosc 2015; 82: $608-614$

[9] Xi Sun, Zhirui Zhou, Jianmin Tian et al. Is single-operator peroral cholangioscopy a useful tool for the diagnosis of indeterminate biliary lesion? A systematic review and meta-analysis Gastrointest Endosc 2015; 82: 79-87

[10] Nguyen NQ, Schoeman MN, Ruszkiewicz A. Clinical utility of EUS before cholangioscopy in the evaluation of difficult biliary strictures. Gastrointest Endosc 2013; 78: 868-874

[11] Tabibian JH, Visrodia KH, Levy MJ et al. Advanced endoscopic imaging of indeterminate biliary strictures. World J Gastrointest Endosc 2015; 7: $1268-1278$

[12] Fuccio L, Hassan C, Laterza L et al. The role of K-ras gene mutation analysis in EUS-guided FNA cytology specimens for the differential diagnosis of pancreatic solid masses: a meta-analysis of prospective studies. Gastrointest Endosc 2013; 78: 596-608 